\title{
Distributed Adaptive Consensus Control of Nonlinear Output-Feedback Systems on Directed Graphs
}

DOI:

10.1016/j.automatica.2016.05.014

\section{Document Version}

Accepted author manuscript

Link to publication record in Manchester Research Explorer

\section{Citation for published version (APA):}

Ding, Z., \& Li, Z. (2016). Distributed Adaptive Consensus Control of Nonlinear Output-Feedback Systems on Directed Graphs. Automatica, 72, 46-52. https://doi.org/10.1016/j.automatica.2016.05.014

\section{Published in:}

Automatica

\section{Citing this paper}

Please note that where the full-text provided on Manchester Research Explorer is the Author Accepted Manuscript or Proof version this may differ from the final Published version. If citing, it is advised that you check and use the publisher's definitive version.

\section{General rights}

Copyright and moral rights for the publications made accessible in the Research Explorer are retained by the authors and/or other copyright owners and it is a condition of accessing publications that users recognise and abide by the legal requirements associated with these rights.

\section{Takedown policy}

If you believe that this document breaches copyright please refer to the University of Manchester's Takedown Procedures [http://man.ac.uk/04Y6Bo] or contact uml.scholarlycommunications@manchester.ac.uk providing relevant details, so we can investigate your claim.

\section{OPEN ACCESS}




\title{
Distributed Adaptive Consensus Control of Nonlinear Output-Feedback Systems on Directed Graphs *
}

\author{
Zhengtao Ding ${ }^{\mathrm{a}}$, Zhongkui Li ${ }^{\mathrm{b}}$ \\ ${ }^{a}$ Control Systems Centre, School of Electrical and Electronic Engineering, University of Manchester, Sackville Street Building, \\ Manchester M13 9PL, UK \\ ${ }^{\mathrm{b}}$ State Key Laboratory for Turbulence and Complex Systems, Department of Mechanics and Serospace Engineering, College of \\ Engineering, Peking University, Beijing 100081, China
}

\begin{abstract}
This paper deals with consensus control in leader-follower format of a class of network-connected uncertain nonlinear systems by output feedback. Each subsystem is in the nonlinear output feedback form with unknown parameters, and the connection graph among the subsystems is directed. Distributed adaptive control inputs are designed to achieve the consensus control in the sense that the subsystem states asymptotically follow the subsystem at node 0 with no input, which is also known as the leader. The proposed adaptive control only uses relative output measurements and the local information of the connection to each subsystem, and hence the proposed adaptive control is fully distributed. The proposed scheme is different from the consensus output regulation schemes literature, and the leader plays a similar role as a reference model in the classic model reference adaptive control.
\end{abstract}

Key words: Adaptive control, Nonlinear systems, Consensus control, Output feedback

\section{Introduction}

For a dynamic system with the subsystems connected by a network, control design of the subsystems can be coordinated to achieve a common control objective by exploring the network connections. The objective for consensus control is to achieve the same subsystem states, often by control design using the relative state or output values of the subsystems in their neighbourhoods. The Laplacian matrix that characterises the connection of a network plays an important role in the consensus control design, and in particular, its second least value of the real parts of the eigenvalues, which is commonly referred to as the connectivity of the network (Fax and Murray, 2004; R.Olfati-Saber and Murray, 2004; OlfatiSaber, Fax and Murray, 2007; Hong, Hu and Gao, 2006). Many proposed design methods for consensus control use Laplacian matrix in the control gain design, and the connectivity is explicitly used in determining the feed-

\footnotetext{
* This paper was not presented at any IFAC meeting. This work was supported in part by the National Natural Science Foundation of China under grants 61473005 and 11332001 , and 111 Project (B08015).

Email addresses: zhengtao.ding@manchester.ac.uk (Zhengtao Ding), zhongkli@pku.edu.cn (Zhongkui Li).
}

back control gains (R.Olfati-Saber and Murray, 2004; Li, Duan, Chen and Huang, 2010; Ding, 2014). With a fixed control gain design, the control inputs only use the relative states or outputs in their neighbourhoods, and this kind of control strategy is often referred to as decentralized. However, the connectivity is a property of an entire network, and it is not an information entirely from the neighbourhood of a subsystem. Therefore, the control design that relies on the information of the Laplacian is not completely distributed.

A distributed scheme was developed for consensus control design based on adaptive control for linear subsystems connected by an undirected graph (Li, Ren, Liu and Xie, 2013). This adaptive scheme is based on the observation that the control structure can be parametrised in such a way that a scalar constant can always be increased in value to achieve consensus control no matter how small the connectivity is. The success of the proposed control in ( $\mathrm{Li}$ et al., 2013) relies on the symmetry in the network connection of an undirected graph. Many adaptive schemes for consensus control requires that the connection graph is undirected to achieve decentralised adaptive laws (Su, Chen, Wang and Lin, 2011; Yu and Xia, 2012; Su and Huang, 2013). For a directed connection graph, the adaptive schemes in (Das and Lewis, 
2010; Zhang and Lewis, 2012) achieved consensus control at a price of sacrificing the asymptotic convergence. Further results on adaptive control under directed communication graph include adaptive coordination of multiple Lagrangian systems (Mei, Ren, Chen and Ma, 2013) and consensus tracking for state-feedback nonlinear systems in the strict feedback form (Wang, Huang, Wen and Fan, 2014), which were obtained using direct local measurements or certain parameters such as $P_{i}$ in (Wang et al., 2014) that requires the knowledge of the Laplacian matrix. The asymmetric connection in a directed graph remained as an obstacle in the extension of adaptive schemes beyond undirected graphs to fully distributed adaptive consensus control, using the relative state or relative output measurements. This obstacle is removed in a recent attempt $(\mathrm{Li}$, Wen, Duan and Ren, 2015) where a careful application of changing supply function is implemented in the Lyapunov function design for the success of distributed adaptive scheme for linear systems with known parameters. Further development is reported in ( $\mathrm{Li}$ and Ding, 2015) for fully distributed adaptive control for linear systems with unknown parameters, and with uncertainty in the network connectivity.

In this paper, we consider the uncertainty in the network connectivity as well as the uncertainty in the subsystem dynamics for a class of nonlinear subsystems. We propose a new distributed adaptive scheme for a class of network-connected nonlinear systems whose subsystems are in the output feedback form and contain unknown parameters. The network connection can be described by a general directed graph as long as there is a directed spanning tree with the node 0 as the root. There are many results on nonlinear and adaptive of nonlinear systems in the output feedback form when they stand alone (Marino and Tomei, 1995; Krstic, Kanellakopoulos and Kokotovic, 1995; Ding, 2013b). It is worth pointing out that the nonlinear output feedback form contains general linear SISO system as a special case, and can be used to describe dynamics of physical systems such at a robot with a flexible joint and a mass-spring-damper system with a nonlinear spring. Consensus control of nonlinear systems in output feedback appeared recently, for example (Ding, 2013a) where consensus output regulation is solved for nonlinear systems in the output feedback form when the parameters are known and the connection graph does not contain circles. The formulation of the problem in this paper is different from the consensus output regulation problem considered in the literature such as (Ding, 2013a; Ding, 2015), where an exosystem with different dynamics from the subsystems is used to generate the desired trajectories. We use one of the subsystems in the node 0 as the leader whose the input is set to zero, and the other subsystems are required to follow the state trajectories of the leader. This leader plays a similar role as the reference model in the traditional model reference adaptive control design. This kind of set-up with the leader having the same dynamics as the subsystems reflects certain applications such as vehicle and robot formation control etc. As the leader, the node 0 does not take information of other subsystems. Due to the extension of the connection graph to a general directed graph which may contain a cycle and the leader-follower structure, the method proposed in (Ding, 2013a; Ding, 2015) would not be applicable even when all the parameters were known. With the nonlinear functions being restricted to polynomials, relative outputs among the subsystems provide a possible signal to achieve consensus control, in a similar way as for the consensus output regulation in (Ding, 2013a), but with a different controller structure. The uncertainty in unknown system parameters are dealt with by adaptive gains in the same spirit as tackling unknown connectivities. However, the nonlinear term in the control input makes the adaptive control scheme shown in $(\mathrm{Li}$ et al., 2015; Li and Ding, 2015) not applicable. In this paper, we propose a new distributed adaptive control scheme to achieve state consensus among the subsystems, via relative output feedback. The proposed adaptive scheme considers the high-order nonlinear terms in the control design, and the stability analysis is carried out with a carefully chosen Lyapunov function. This distributed adaptive scheme tackles the unknown connectivity and unknown parameters in the nonlinear subsystems simultaneously. An example is included to demonstrate the proposed distributed adaptive scheme for consensus control.

\section{Problem Statement}

In this paper, we consider a set of $N+1$ nonlinear subsystems, of which the subsystems are described by

$\dot{x}_{i}=A_{c} x_{i}+\phi\left(y_{i}\right)+b u_{i}$,

$y_{i}=C x_{i}$,

with $b, C^{T} \in \mathbb{R}^{n}$ and

$A_{c}=\left[\begin{array}{ccccc}0 & 1 & 0 & \ldots & 0 \\ 0 & 0 & 1 & \ldots & 0 \\ \vdots & \vdots & \vdots & \ddots & \vdots \\ 0 & 0 & 0 & \ldots & 1 \\ 0 & 0 & 0 & \ldots & 0\end{array}\right]$,

$b=\left[\begin{array}{c}b_{1} \\ \vdots \\ b_{n}\end{array}\right], \quad C^{T}=\left[\begin{array}{c}1 \\ 0 \\ \vdots \\ 0\end{array}\right]$, 
for $i=0,1, \ldots N$, where $x_{i} \in \mathbb{R}^{n}$ is the state vector, with $n$ a known positive constant integer denoting the order of the subsystems, $y_{i}, u_{i} \in \mathbb{R}$ are the output and input respectively of the $i$ th subsystem, $b$ is an unknown Hurwitz vector with $b_{1} \neq 0$, which implies the relative degree of the system is $1, \phi: \mathbb{R} \times \mathbb{R}^{m} \rightarrow \mathbb{R}^{n}$ contains unknown nonlinear functions with each element being polynomials of its variables and satisfies $\phi(0)=0$. Note that we start the index from 0 for notational convenience in the presentation later.

The connections among the subsystems are specified by a directed graph $\mathcal{G}$ which consists of a set of vertices denoted by $\mathcal{V}$ and a set of edges denoted by $\mathcal{E}$. A vertex represents a subsystem, and each edge represents a connection. Associated with the graph, its adjacency matrix $A$ with elements $a_{i j}$ denotes the connections such that $a_{i j}=1$ if there is a path from subsystem $j$ to subsystem $i$, and $a_{i j}=0$ otherwise. We define the Laplacian matrix $L$ in the normal way as $l_{i i}=\sum_{j=1}^{N} a_{i j}$ and $l_{i j}=-a_{i j}$ when $i \neq j$.

We take the subsystem at node $i=0$, or the root node, as the leader whose input $u_{0}$ is set to 0 . We define the output tracking errors as

$e_{i}=y_{i}-y_{0}$

for $i=1, \ldots, N$.

The distributed adaptive consensus control problem considered in this paper is to design an adaptive control strategy using the relative output information $y_{i}-y_{j}$, $i \neq j$, provided by the network connection to each subsystem to ensure the state consensus in the sense that the subsystem states asymptotically follow the state of the leader, i.e., the subsystem at node 0 , under any initial condition of the system in the state space, which implies the convergence to 0 of output tracking errors $e_{i}$ for $i=1, \ldots, N$.

We make three assumptions about the dynamics of the subsystems and the connections between the subsystems.

Assumption 1. The sign of the high-frequency gain $b_{1}$ is known.

Assumption 2. For the nonlinear function $\phi$, the following condition holds:

$\left\|\phi\left(y_{i}\right)-\phi\left(y_{0}\right)\right\|^{2} \leq \gamma_{\phi}\left(e_{i}^{2}+e_{i}^{2 p}\right)$

where $\gamma_{\phi}$ is an unknown positive real number, and $p$ is a known positive integer.

Assumption 3. The connection graph contains a directed spanning tree with node 0 as the root.
Remark 1 The subsystem (1) is in the standard nonlinear output feedback form. The geometric conditions are specified in (Krstic et al., 1995) for a general nonlinear system that can be transformed to the output feedback form. It contains general linear SISO systems with completely unknown parameters as a special case.

Remark 2 Examples for physical systems in the output feedback form are discussed in some details in (Marino and Tomei, 1995), which include the systems such as a robot with a flexible joint etc. From the practical point of view, nonlinear functions can be approximated by polynomials in certain regions, and we would expect that the control design for the output feedback forms with nonlinear functions in polynomials to be applicable to the systems in the standard output feedback form. Some common physical systems such as mass-spring-damper systems with hardened or softened springs can be modelled in the nonlinear output feedback form with polynomial nonlinearities for the nonlinear relations between the spring forces and the displacements.

Remark 3 Assumption 2 is clearly satisfied for linear systems with unknown parameter. It is also satisfied when the tracking trajectory $y_{0}$ is bounded and the polynomial order is up to p. Physical systems such as mass-springdamper systems and van der Pol oscillators satisfy Assumptions 1 and 2. Note that a van der Pol oscillator describes the dynamics of a RLC circuit with a nonlinear resistor (Ding, 2013b). In practice, the leader's trajectory is expected to be bounded, and the other nonlinear functions can be approximated by polynomials. In such a case, Assumption 2 is then satisfied.

\section{Preliminary Results}

We introduce a state transformation to extract the internal dynamics of (1) with $\bar{z}_{i} \in \mathbb{R}^{n-1}$ given by

$\bar{z}_{i}=x_{i, 2: n}-\frac{b_{2: n}}{b_{1}} y_{i}$

where $(\cdot)_{2: n}$ refers to the vector or matrix formed by the 2 nd row to the $n$th row. With the coordinates $\left(\bar{z}_{i}, y_{i}\right)$, (1) is rewritten as

$\dot{\bar{z}}_{i}=B \bar{z}_{i}+\bar{\phi}\left(y_{i}\right)$

$\dot{y}_{i}=\bar{z}_{i, 1}+\bar{\phi}_{y}\left(y_{i}\right)+b_{1} u_{i}$, 
where $B$ is the left companion matrix of $b$ given by

$B=\left[\begin{array}{cccc}-b_{2} / b_{1} & 1 & \ldots & 0 \\ -b_{3} / b_{1} & 0 & \ddots & 0 \\ \vdots & \vdots & \vdots & \vdots \\ -b_{n-1} / b_{1} & 0 & \ldots & 1 \\ -b_{n} / b_{1} & 0 & \ldots & 0\end{array}\right]$,

and

$$
\begin{aligned}
\bar{\phi}\left(y_{i}\right) & =B \frac{b_{2: n}}{b_{1}} y_{i}+\phi_{2: n}\left(y_{i}\right)-\frac{b_{2: n}}{b_{1}} \phi_{1}\left(y_{i}\right), \\
\bar{\phi}_{y}\left(y_{i}\right) & =\frac{b_{2}}{b_{1}} y_{i}+\phi_{1}\left(y_{i}\right) .
\end{aligned}
$$

Let $z_{i}=\bar{z}_{i}-\bar{z}_{0}$. The dynamics of $\left(z_{i}, e_{i}\right)$ are obtained as

$\dot{z}_{i}=B z_{i}+\psi\left(y_{i}, y_{0}\right)$,

$\dot{e}_{i}=h^{T} z_{i}+\psi_{y}\left(y_{i}, y_{0}\right)+b_{1} u_{i}$,

where $h=[1,0, \ldots, 0]^{T} \in \mathbb{R}^{n-1}, \psi\left(y_{i}, y_{0}\right)=\bar{\phi}\left(y_{i}\right)-$ $\bar{\phi}\left(y_{0}\right)$ and $\psi_{y}\left(y_{i}, y_{0}\right)=\bar{\phi}_{y}\left(y_{i}\right)-\bar{\phi}_{y}\left(y_{0}\right)$.

To deal with polynomials, we have the following result, which can be obtained from Young's inequality.

Lemma 1 For a variable $y \in \mathbb{R}>0$, the following inequality holds:

$y^{r_{1}} \leq \mu_{1} y^{r_{2}}+\mu_{2} y^{r_{3}}$

where $r_{1}, r_{2}$ and $r_{3}$ are positive integers with $r_{3}<r_{1}<r_{2}$ and $r_{1} \geq 2$, and $\mu_{1}$ and $\mu_{2}$ are positive real constants. Furthermore, with $\mu_{1}$ chosen, $\mu_{2}$ is a function of $\mu_{1}$ and $r_{i}$ s for $i=1,2,3$, and is independent of $y$.

Proof. Let $a, b>0$ and $a+b=r_{1}$. Let $p, q>0$ and $\frac{1}{p}+\frac{1}{q}=1$. From Young's inequality, we have

$\left(\mu y^{a}\right)\left(y^{b} \mu^{-1}\right) \leq \mu^{p} \frac{y^{a p}}{p}+\mu^{-q} \frac{y^{b q}}{q}$

where $\mu$ is any positive number. With $a p=r_{2}$ and $b q=$ $r_{3}$, we have

$a=\frac{r_{2}\left(r_{1}-r_{3}\right)}{r_{2}-r_{3}}$,

$b=\frac{r_{3}\left(r_{2}-r_{1}\right)}{r_{2}-r_{3}}$,

$p=\frac{r_{2}-r_{3}}{r_{1}-r_{3}}$,

$q=\frac{r_{2}-r_{3}}{r_{2}-r_{1}}$.
Setting $\mu^{p} / p=\mu_{1}$, it can be obtained that

$\mu_{2}=\frac{r_{2}-r_{1}}{r_{2}-r_{3}}\left(\frac{r_{2}-r_{3}}{r_{1}-r_{3}} \mu_{1}\right)^{-\frac{r_{1}-r_{3}}{r_{2}-r_{1}}}$.

From Assumption 2 and applying the result shonw in Lemma 1 , it is easy to obtain that

$$
\begin{aligned}
\left\|\psi\left(y_{i}, y_{0}\right)\right\|^{2} & \leq \gamma_{\psi}\left(e_{i}^{2}+e_{i}^{2 p}\right), \\
\psi_{y}^{2}\left(y_{i}, y_{0}\right) & \leq \gamma_{y}\left(e_{i}^{2}+e_{i}^{2 p}\right),
\end{aligned}
$$

for some unknown positive real constants $\gamma_{\psi}$ and $\gamma_{y}$.

We have the following results before the presentation of control design.

Lemma 2 Based on Assumption 3, with the Laplacian matrix being partitioned as

$L=\left[\begin{array}{cc}0 & 0_{1 \times N} \\ L_{0 c} & Q\end{array}\right]$,

the matrix $Q \in \mathbb{R}^{N \times N}$ is a non-singular M-matrix ( $Q u$, 2009), and there exists a positive diagonal matrix $G=$ $\operatorname{diag}\left\{g_{1}, \ldots, g_{N}\right\}$ such that

$G Q+Q^{T} G \geq r_{0} I$

for some positive constant $r_{0}$.

Proof. This result has been used in literature such as $(\mathrm{Li}$ et al., 2015). It is easy to see that $Q$ is a non-singular M-matrix from the structure of $L(\mathrm{Qu}, 2009)$, and the existence of $G$ for (12) follows from a theorem in $(\mathrm{Qu}$, 2009) .

Note that $q_{i j}=l_{i j}$ for $i, j=1, \ldots, N$. We define, for $i=1, \ldots, N$,

$\zeta_{i}=\sum_{j=0}^{N} a_{i j}\left(y_{i}-y_{j}\right)$.

It can be obtained that

$$
\begin{aligned}
\zeta_{i} & =\sum_{j=1}^{N} l_{i j} y_{j}+l_{i 0} y_{0} \\
& =\sum_{j=1}^{N} l_{i j} y_{j}-\sum_{j=1}^{N} l_{i j} y_{0} \\
& =\sum_{j=1}^{N} q_{i j} e_{j}
\end{aligned}
$$


or in the vector form, $\zeta=Q e$ where $\zeta, e \in \mathbb{R}^{N}$ are the vectors with $\zeta_{i}$ and $e_{i}$ as elements, respectively. A useful relationship between $e$ and $\zeta$ is shown in the following lemma.

Lemma 3 With $\zeta=Q e$, the following inequality holds for any positive integer $p$,

$\sum_{i=1}^{N} e_{i}^{2 p} \leq N^{p-1} \sigma^{2 p}(Q) \sum_{i=1}^{N} \zeta_{i}^{2 p}$

where $\sigma(Q)$ denotes the singular value of $Q$.

Proof. A direct evaluation gives

$$
\begin{aligned}
\sum_{i=1}^{N} \zeta_{i}^{2 p} & =N\left(\left[\frac{1}{N} \sum_{i=1}^{N}\left(\zeta_{i}^{2}\right)^{p}\right]^{1 / p}\right)^{p} \\
& \geq N\left(\left[\frac{1}{N} \sum_{i=1}^{N}\left(\zeta_{i}^{2}\right)\right]\right)^{p} \\
& =N^{1-p}\left(\|\zeta\|^{2}\right)^{p} \\
& =N^{1-p}\|Q e\|^{2 p} \\
& \geq N^{1-p} \sigma^{-2 p}(Q)\|e\|^{2 p} \\
& \geq N^{1-p} \sigma^{-2 p}(Q)\left(\sum_{i=1}^{N} e_{i}^{2}\right)^{p} \\
& \geq N^{1-p} \sigma^{-2 p}(Q) \sum_{i=1}^{N} e_{i}^{2 p}
\end{aligned}
$$

from which (14) is obtained.

\section{Control Design}

We design the control input as

$u_{i}=-\operatorname{sign}\left(b_{1}\right)\left(k_{i}+\rho_{i}\right)\left(\zeta_{i}+\zeta_{i}^{2 p-1}\right)$

where $\rho_{i}=\zeta_{i}^{2}$ and $k_{i}$ is generated by

$\dot{k}_{i}=\gamma_{k}\left(\zeta_{i}^{2}+\zeta_{i}^{2 p}\right)$ with $k_{i}(0)=k_{0}$

with $k_{0}$ any known positive constant. Note that $k_{i}$ can be viewed as an adaptive gain.

Theorem 4 For the network connected nonlinear systems with subsystem dynamics (1), the control input (15) together with the adaptive law (16) solve the distributed adaptive consensus control problem under Assumptions 1, 2 and 3.

Proof. The closed-loop subsystem dynamics of $e_{i}$ can be obtained as

$\dot{e}_{i}=h^{T} z_{i}+\psi_{y}\left(y_{i}, y_{0}\right)-\left|b_{1}\right|\left(k_{i}+\rho_{i}\right)\left(\zeta_{i}+\zeta_{i}^{2 p-1}\right)$.
Let

$$
\begin{aligned}
V_{\zeta}= & \sum_{i=1}^{N} 2 g_{i}\left(k_{i}\left(\frac{\zeta_{i}^{2}}{2}+\frac{\zeta_{i}^{2 p}}{2 p}\right)+\left(\frac{\zeta_{i}^{4}}{4}+\frac{\zeta_{i}^{2 p+2}}{2 p+2}\right)\right) \\
& +\frac{1}{2 \gamma_{k}} \sum_{i=1}^{N}\left(k_{i}-k^{*}\right)^{2}
\end{aligned}
$$

where $k^{*}$ is a constant, to be determined later. Using (17) and (16), we have

$$
\begin{aligned}
\dot{V}_{\zeta}= & \sum_{i=1}^{N} 2 g_{i}\left(k_{i}+\rho_{i}\right)\left(\zeta_{i}+\zeta_{i}^{2 p-1}\right) \sum_{j=1}^{N} q_{i j} \dot{e}_{j} \\
& +\sum_{i=1}^{N} g_{i}\left(\zeta_{i}^{2}+\frac{\zeta_{i}^{2 p}}{p}\right) \dot{k}_{i}+\frac{1}{\gamma_{k}} \sum_{i=1}^{N}\left(k_{i}-k^{*}\right) \dot{k}_{i} \\
= & -\left|b_{1}\right| \zeta^{T}(K+\rho)\left(I_{N}+\rho^{p-1}\right)\left(G Q+Q^{T} G\right) \\
& \times\left(I_{N}+\rho^{p-1}\right)(K+\rho) \zeta \\
& +2 \zeta^{T}(K+\rho)\left(I_{N}+\rho^{p-1}\right) G Q\left(\left(I_{N} \otimes h^{T}\right) z+\Psi_{y}\right) \\
& +\gamma_{k}\left(\rho+\rho^{p} / p\right) G\left(\rho+\rho^{p}\right)+\zeta^{T} K\left(I_{N}+\rho^{p-1}\right) \zeta^{N} \\
& -k^{*} \sum_{i=1}^{N}\left(\zeta_{i}^{2}+\zeta_{i}^{2 p}\right)
\end{aligned}
$$

where $g_{i}, i=1, \ldots, N$, are the diagonal elements of $G$ defined in (12), $z$, and $\Psi_{y}$ are vectors that are formed by stacking up their corresponding individual elements $z_{i}$ and $\psi_{y}\left(y_{i}, y_{0}\right)$ in the order from 1 to $N$, respectively, and $K=\operatorname{diag}\left\{k_{i}\right\}, \rho=\operatorname{diag}\left\{\rho_{i}\right\}$.

From (12) and Young's inequality, we have

$$
\begin{aligned}
\dot{V}_{\zeta} \leq & -\frac{5}{8} r\left\|(K+\rho)\left(I_{N}+\rho^{p-1}\right) \zeta\right\|^{2} \\
& +\frac{8}{r}\left(\|G Q\|^{2}\|z\|^{2}+\|G Q\|^{2}\left\|\Psi_{y}\right\|^{2}+\frac{1}{4}\|\zeta\|^{2}\right) \\
& +\gamma_{k}\left(\rho+\rho^{p} / p\right) G\left(\rho+\rho^{p}\right) \\
& -k^{*} \sum_{i=1}^{N}\left(\zeta_{i}^{2}+\zeta_{i}^{2 p}\right)
\end{aligned}
$$

where $r=\left|b_{1}\right| r_{0}$, and we used

$$
\begin{aligned}
& \zeta^{T} K\left(I_{N}+\rho^{p-1}\right) \zeta \\
\leq & \frac{r}{8}\left\|(K+\rho)\left(I_{N}+\rho^{p-1}\right) \zeta\right\|^{2}+\frac{2}{r}\|\zeta\|^{2} .
\end{aligned}
$$

Observe that

$$
\left\|(K+\rho)\left(I_{N}+\rho^{p-1}\right) \zeta\right\|^{2} \geq \rho^{3}\left(I_{N}+\rho^{p-1}\right)^{2}
$$

whose highest power of $\rho$ is $2 p+1$, and the highest power in the terms in the polynomial $\gamma_{k}\left(\rho+\rho^{p} / p\right) G\left(\rho+\rho^{p}\right)$ 
is $2 p$. Since all the matrices are diagonal, we can apply Lemma 1 element-wise. Hence, from Lemma 1, we establish that

$$
\begin{aligned}
& \gamma_{k}\left(\rho+\rho^{p} / p\right) G\left(\rho+\rho^{p}\right) \\
\leq & \frac{r}{8}\left\|(K+\rho)\left(I_{N}+\rho^{p-1}\right) \zeta\right\|^{2}+\mu(r) \rho
\end{aligned}
$$

where $\mu: \mathbb{R}^{+} \rightarrow \mathbb{R}^{+}$is a function that depends on unknown parameters.

From (11) and Lemma 3, it can be shown that there exists a positive real constant $\mu_{y}$ such that

$\left\|\Psi_{y}\right\|^{2} \leq \gamma_{y} \sum_{i=1}^{N}\left(e_{i}^{2}+e_{i}^{2 p}\right) \leq \mu_{y} \sum_{i=1}^{N}\left(\zeta_{i}^{2}+\zeta_{i}^{2 p}\right)$.

From (19), (20) and (18), we obtain

$$
\begin{aligned}
\dot{V}_{\zeta} \leq & -\frac{r}{2}\left\|(K+\rho)\left(I_{N}+\rho^{p-1}\right) \zeta\right\|^{2}+\frac{8}{r}\|G Q\|^{2}\|z\|^{2} \\
& -\left(k^{*}-\mu(r)-\|G Q\|^{2} \mu_{y}-\frac{2}{r}\right) \sum_{i=1}^{N}\left(\zeta_{i}^{2}+\zeta_{i}^{2 p}\right)
\end{aligned}
$$

Since $B$ is Hurwitz, there exists a positive definite matrix $P$ such that

$P B+B^{T} P=-3 I$.

Let

$V_{z}=\sum_{i=1}^{N} z_{i}^{T} P z_{i}$

From (6), it can be obtained that

$$
\begin{aligned}
\dot{V}_{z} & =-3 \sum_{i=1}^{N}\left\|z_{i}\right\|^{2}+2 \sum_{i=1}^{N} z_{i}^{T} P \psi\left(y_{i}, y_{0}\right) \\
& \leq-2\|z\|^{2}+\|P\|^{2} \sum_{i=1}^{N}\left\|\psi\left(y_{i}, y_{0}\right)\right\|^{2} \\
& \leq-2\|z\|^{2}+\|P\|^{2} \mu_{\psi} \sum_{i=1}^{N}\left(\zeta_{i}^{2}+\zeta_{i}^{2 p}\right)
\end{aligned}
$$

where $\mu_{\psi}$ is a positive real constant. To obtain the last line in (22), we have used (10) and Lemma 3.

Finally, let

$V=V_{\zeta}+\frac{8}{r}\|G Q\|^{2} V_{z}$
From (21) and (22), we have

$$
\begin{aligned}
\dot{V} \leq & -\frac{r}{2}\left\|(K+\rho)\left(I_{N}+\rho^{p-1}\right) \zeta\right\|^{2}-\frac{8}{r}\|G Q\|^{2}\|z\|^{2} \\
- & \left(k^{*}-\mu(r)-\|G Q\|^{2} \mu_{y}-\frac{2}{r}\right. \\
& \left.-\frac{8}{r}\|G Q\|^{2} \mu_{\psi}\|P\|^{2}\right) \sum_{i=1}^{N}\left(\zeta_{i}^{2}+\zeta_{i}^{2 p}\right) .
\end{aligned}
$$

Setting

$k^{*}=\mu(r)+\|G Q\|^{2} \mu_{y}+\frac{2}{r}+\frac{8}{r}\|G Q\|^{2} \mu_{\psi}\|P\|^{2}$

results in

$\dot{V} \leq-\frac{r}{2}\left\|(K+\rho)\left(I_{N}+\rho^{p-1}\right) \zeta\right\|^{2}-\frac{8}{r}\|G Q\|^{2}\|z\|^{2}$.

Therefore we can conclude that the variables $\zeta, z$ and $K$ are bounded and $\zeta, z \in L_{2 \cap \infty}$. From the boundedness of their derivatives and Babalat's lemma, we have $\lim _{t \rightarrow \infty} z(t)=0$ and $\lim _{t \rightarrow \infty} \zeta(t)=0$ which implies that $\lim _{t \rightarrow \infty} e_{i}(t)=0$ for $i=1, \ldots, N$.

Remark 4 In the above proof, the boundedness of $k_{i} s$ in $K$ is established via the boundedness of $V$, as the Lyapunov function $V$ is positive definite respect to the variables $k_{i}$. Intuitively, if we integrate both sides of (16), we have

$$
\begin{aligned}
k_{i} & =k_{0}+\gamma_{k}\left(\int_{0}^{t} \zeta_{i}^{2}(t) d t+\int_{0}^{t} \zeta_{i}^{2 p}(t) d t\right) \\
& \leq k_{0}+\gamma_{k}\left(1+\left\|\zeta_{i}\right\|_{\infty}^{2(p-1)}\right)\left\|\zeta_{i}\right\|_{2}^{2},
\end{aligned}
$$

where $\left\|\zeta_{i}\right\|_{\infty}$ and $\left\|\zeta_{i}\right\|_{2}$ denote the functional norms of $\zeta_{i}$ in $L_{\infty}$ and $L_{2}$ respectively. Hence, the boundedness of $k_{i}$ follows the fact $\zeta_{i} \in L_{2 \cap \infty}$. The boundedness of $u_{i}$ in (15) then follows from the boundedness of $k_{i}$ and $\zeta_{i}$.

\section{Example}

In this section, we will show some details of the proposed consensus control design through an example. The system under consideration is a connection of 5 subsystems, each of them is described by a second-order state space model as

$\dot{x}_{i}=\left[\begin{array}{ll}0 & 1 \\ 0 & 0\end{array}\right] x_{i}+\left[\begin{array}{c}\mu\left(y_{i}-\frac{1}{3} y_{i}^{3}\right) \\ -y_{i} / \mu\end{array}\right]+\left[\begin{array}{l}b_{1} \\ b_{2}\end{array}\right] u_{i}$

with $y_{i}=x_{i, 1}$, where $\mu, b_{1}$ and $b_{2}$ are unknown positive real parameters. Note that when $u_{i}=0$, the system is a 
van der Pol oscillator, and its trajectories are bounded. It is also worth noting that a van der Pol oscillator describes the dynamics of a RLC circuit with a nonlinear resistor. Hence, it can be shown that Assumption 2 is satisfied with $p=3$.

The adjacency matrix is given by

$A=\left[\begin{array}{lllll}0 & 0 & 0 & 0 & 0 \\ 1 & 0 & 0 & 1 & 0 \\ 0 & 1 & 0 & 0 & 0 \\ 1 & 0 & 0 & 0 & 1 \\ 0 & 0 & 1 & 0 & 0\end{array}\right]$

and the resultant Laplacian matrix is obtained as

$L=\left[\begin{array}{ccccc}0 & 0 & 0 & 0 & 0 \\ -1 & 2 & 0 & 1 & 0 \\ 0 & -1 & 1 & 0 & 0 \\ -1 & 0 & 0 & 2 & -1 \\ 0 & 0 & -1 & 0 & 1\end{array}\right]$

Since $b_{1}>0$, Assumption 1 is satisfied. From the network connection, it can be shown that the networkconnected system with the subsystem (25) also satisfies Assumptions 3. The control inputs are designed as, for $i=1, \ldots, 5$,

$u_{i}=-\left(k_{i}+\rho_{i}\right)\left(\zeta_{i}+\zeta_{i}^{5}\right)$,

$\dot{k}_{i}=\gamma_{k}\left(\zeta_{i}^{2}+\zeta_{i}^{6}\right)$.

Simulation study has been carried out with the parameters $b_{1}=b_{2}=1$ and $\gamma_{k}=5$. The parameter $\mu$ is set as

$\mu=\left\{\begin{array}{c}0.5 \text { for } 0 \leq t \leq 20 \\ 2 \text { for } t>20\end{array}\right.$

so that two different limit cycles are used as the trajectories of the leader.

The simulation results of the subsystem states are shown in Figures 1 and 2. Figures 3 and 4 show the subsystem inputs and the gains $k_{i}$. It can be seen that both the output and the states converge to common trajectories of the leader, which are shown in Figures 1 and 2 with the initial values $[2.0-1.0]^{T}$. It is also noted that that the trajectories are different after 20 seconds in the simulation, due to the change in the value of $\mu$.

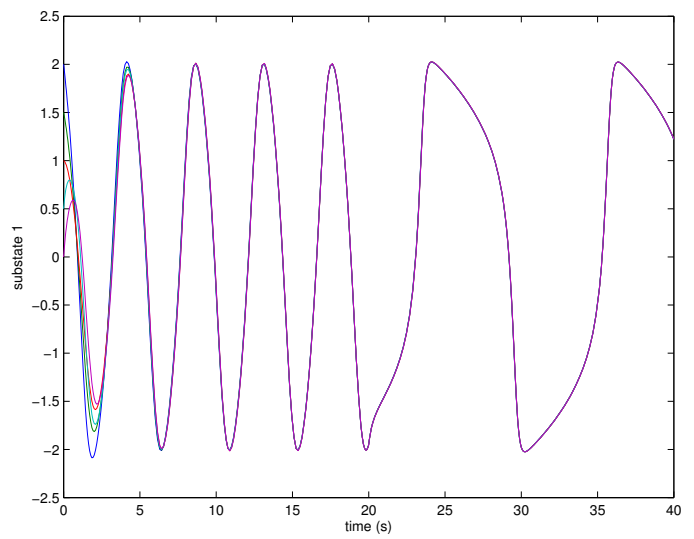

Fig. 1. The subsystem states $x_{i, 1}$, i.e., the outputs.

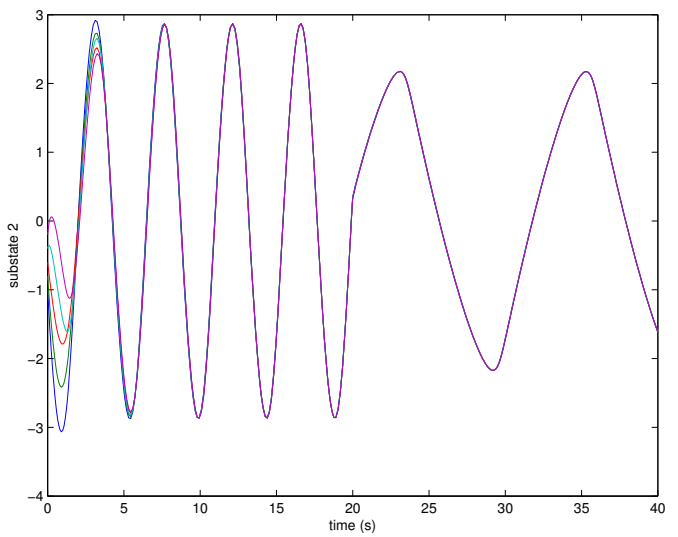

Fig. 2. The subsystem states $x_{i, 2}$.

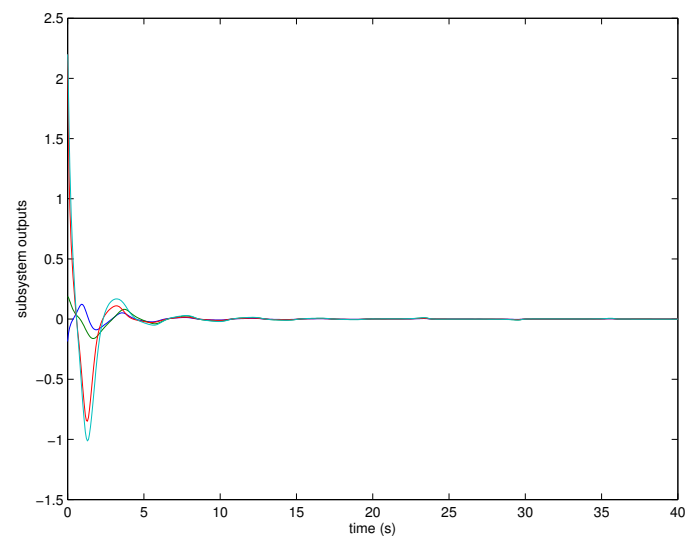

Fig. 3. The subsystem inputs.

\section{Conclusions}

We have proposed a distributed adaptive scheme for consensus control of a network-connected nonlinear sys- 


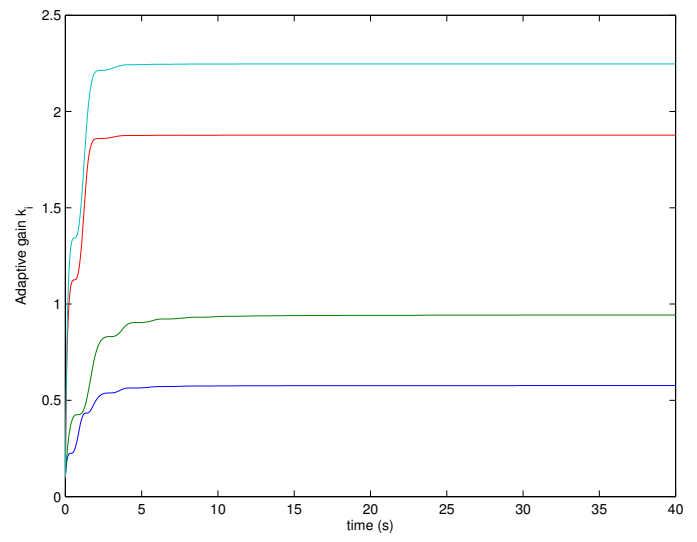

Fig. 4. The controller gains $k_{i}$.

tems via relative output feedback. The proposed scheme does not use any global information, not even the connectivity of the directed network connection. Adaptive control techniques are used to tackle both the unknown connectivity and the unknown parameters in the subsystem dynamics. This proposed control design is different from consensus output regulation, and the leader acts as a reference model. Comparing with the existing results on distributed adaptive schemes, this result extends the distributed adaptive scheme to nonlinear systems and uncertain systems, including uncertain linear systems. Further research could be carried out to relax some of the assumptions made in the proposed design. One direction would be to allow subsystems to have different dynamics. It is not expected to be straightforward for the class of the systems considered here, at it may involve some obstacles that people would encounter in nonlinear output regulation with nonlinear exosystems. There is a good chance to extend the results for uncertain linear subsystems with a possible exploration of robust adaptive control techniques.

\section{References}

Das, A. and Lewis, F. (2010), 'Distributed adaptive control for synchronization of unknown nonlinear networked systems', Automatica 46, 2014-2021.

Ding, Z. (2013a), 'Consensus output regulation of a class of heterogeneous nonlinear systems', IEEE Trans. Automatic Control 58(10), 2648-2653.

Ding, Z. (2013b), Nonlinear and Adaptive Control Systems, IET, London.

Ding, Z. (2014), 'Consensus control of a class of lipschitz nonlinear systems', Int. J. Control 87(11), 2372-2382.

Ding, Z. (2015), 'Adaptive consensus output regulation of a class of nonlinear systems with unknown high-frequency gain', Automatica 51, 348-355.

Fax, J. A. and Murray, R. M. (2004), 'Information flow and cooperative control of vehicle formation', IEEE Trans. Automa. Contr. 49(9), 1465-1476.
Hong, Y., Hu, J. and Gao, L. (2006), 'Tracking control for multiagent consensus with an active leader and variable topology', Automatica 42(7), 1177-1182.

Krstic, M., Kanellakopoulos, I. and Kokotovic, P. V. (1995), Nonlinear and Adaptive Control Design, John Wiley \& Sons, New York.

Li, Z. and Ding, Z. (2015), 'Distributed adaptive consensus and output tracking of unknown linear systems on directed graphs', Automatica 55, 12-18.

Li, Z., Duan, Z., Chen, G. and Huang, L. (2010), 'Consensus of multiagent systems and synchronization of complex networks, a unified viewpoint', IEEE Trans. Circuits Syst. I. 57(1), 213-224.

Li, Z., Ren, W., Liu, X. and Xie, L. (2013), 'Distributed consensus of linear multi-agent systems with adaptive dynamic protocols', Automatica 49(7), 1986-1995.

Li, Z., Wen, G., Duan, Z. and Ren, W. (2015), 'Designing fully distributed consensus protocols for linear multi-agent systems with directed graphs', IEEE Trans. Automa. Contr. 60(4), 1152-1157.

Marino, R. and Tomei, P. (1995), Nonlinear Control Design: Geometric, Adaptive, and Robust, Prentice-Hall, London.

Mei, J., Ren, W., Chen, J. and Ma, G. (2013), 'Distributed adaptive coordination for multiple lagrangian systems under a directed graph without using neighbors velocity information', Automatica 49, 1723-1731.

Olfati-Saber, R., Fax, J. A. and Murray, R. M. (2007), 'Consensus and coop- eration in networked multi-agent systems', Proceedings of the IEEE 95(1), 215-233.

$\mathrm{Qu}$, Z. (2009), Cooperative Control of Dynamical Systems, Springer-Verlag, London.

R.Olfati-Saber and Murray, R. M. (2004), 'Consensus problems in networks of agents with switching topology and time-delay', IEEE Trans. Automa. Contr. 49(9), 1520-1533.

$\mathrm{Su}, \mathrm{H}$., Chen, G., Wang, X. and Lin, Z. (2011), 'Adaptive secondorder consensus of networked mobile agents with nonlinear dynamics', Automatica 47(2), 368-375.

$\mathrm{Su}$, Y. and Huang, J. (2013), 'Cooperative adaptive output regulation for a class of nonlinear uncertain multi-agent systems with unknown leader', Systems \& Control Letters 62(6), 461-467.

Wang, W., Huang, J., Wen, C. and Fan, H. (2014), 'Distributed adaptive control for consensus tracking with application to formation control of nonholonomic mobile robots', Automatica 50, 1254-1263.

Yu, H. and Xia, X. (2012), 'Adaptive consensus of multi-agents in networks with jointly connected topologies', Automatica 48, 1783-1790.

Zhang, H. and Lewis, F. (2012), 'Adaptive cooperative tracking control of high-order nonlinear systems with unknown dynamics', Automatica 48, 1432-1439. 